\section{Action of Oxalyl Chloride on the Aromatic Hydrocarbons}

THE action of oxalyl chloride on an aromatic hydrocarbon in the presence of anhydrous aluminium chloride and in a solvent (for example, carbon disulphide) can lead to the production of the aromatic acid as the chief reaction product. This may provide, too, a general method for the synthesis of $p$-alkylbenzoic acids ${ }^{1}$.

Schönberg and Kraemer ${ }^{2}$ isolated $p$-ethylbenzoic acid only by the interaction of oxalyl chloride, ethylbenzene, and aluminium chloride in carbon disulphide solution at a low temperature. No mention was made of the yield of the acid. On repeating their experiment and modifying the procedure so as to extract the acid directly from the reacting mixture with a dilute alkali solution, $p$-ethylbenzoic acid (m.p. 112-113 ${ }^{\circ}$ was obtained in 43 per cent yield, rising to 66 per cent if equimolecular amounts of the substances interacted at room temperature. $p, p^{\prime}$-Diethyldiphenyl ketone was also isolated.

The action of oxalyl chloride on the higher $n$-alkylbenzenes, under similar conditions, led to the formation of the corresponding $p$-n-alkylbenzoic acids in satisfactory yield. Results are shown in the accompanying table.

Action of oxalyl chloride on alkylbenzenes in carbon disulphide in presence of aluminium chloride at room temperature

\begin{tabular}{|l|l|c|}
\hline \multicolumn{1}{|c|}{ Alkylbenzene } & \multicolumn{1}{|c|}{ Acid formed } & Yield of acid (\%) \\
\hline Toluene & $p$-toluic & 60 \\
Ethylbenzene & $p$-ethylbenzoic & 66 \\
$n$-propylbenzene & $p$-n -propylbenzoic & 70 \\
$n$-butylbenzene & $p$ - $n$-butylbenzoic & 50 \\
$n$-amylbenzene & $p-n$-amylbenzoic & 50 \\
$n$-hexylbenzene & $p$-n -hexylbenzoic & 40 \\
$n$-heptylbenzene & $p$-n-heptylbenzoic & 40 \\
$n$-octylbenzene & $p$-n-octyibenzoic & 3 \\
\hline
\end{tabular}

The yield of the $n$-octylbenzoic acid was exceptionally low. This might be due to the fact that the reaction was not complete at room temperature.

Chemistry Department,

Faculty of Science,

Fouad I University,

Abbassia, Cairo.

May 4.

Comp. Zaki and Fahim, J. Chem. Soc., 307 (1942).

ser., 55, 1174 (1922).

\section{Selective Development of Fission Tracks in an Electron-sensitive Emulsion}

THE interpretation of records of nuclear reactions occurring in photographic emulsions is materially assisted by differences in the solidity and grainfrequency of tracks produced by particles of different ionizing power ${ }^{\mathbf{1}}$. Discrimination between tracks of various particles can be enhanced by using emulsions with a range of intrinsic sensitivities ${ }^{2}$; to a lesser extent by varying development time and composition of developer ${ }^{2,3}$; or by applying oxidizing solutions between exposure and development ${ }^{4}$.

Special emulsions have been produced which are sufficiently sensitive to record tracks of electrons $s^{5,6}$, whereas at the other end of the range there are emulsions sensitive only to tracks of fission fragments ${ }^{7,8}$. The advantage of the latter is, of course,

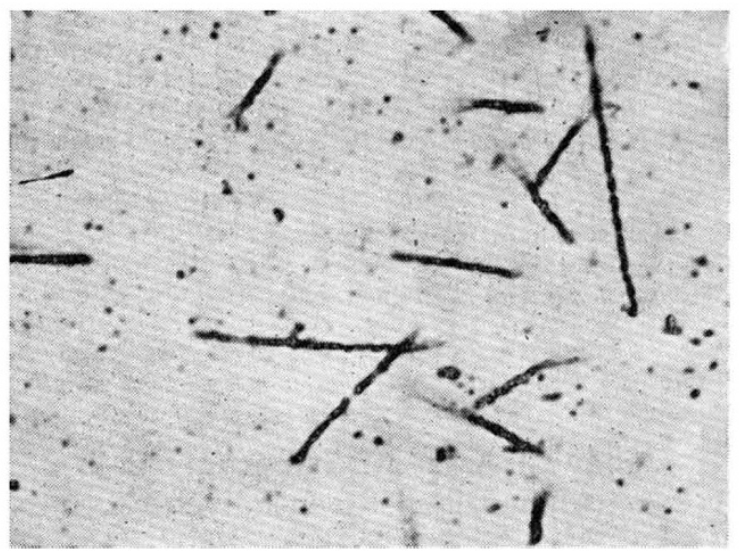

Fig. 1. Photomicrograph of tracks from uranium layer, dipping into NT. $2 a$ emulsion. Development $10 \mathrm{~min}$. D.19b

that fission fragments alone can be recorded in spite of a heavy background of radiation from other less strongly ionizing particles.

It would be very convenient to be able to utilize an electron-sensitive emulsion for the selective recording of fission tracks, so that this whole range of results could be spanned with a single emulsion. A means of achieving this result by controlled under-development is the subject of the present communication.

Deliberate under-development implies that the development process will be stopped before all the exposed emulsion grains have been developed. Control of the results (particularly with thickly coated emulsions) is therefore facilitated by the use of long development times with severely restrained and slowworking developers. In preliminary tests it was noticed that a para-aminophenol solution, used at such a low $p H$ that it would not even develop alphaparticle tracks, still gave strong development of abrasion marks in the emulsion surface. It seemed possible that such a solution would also develop fission tracks.

Glass plates were coated with a suspension of ammonium diuranate in gelatin (equivalent to $0.01 \mathrm{gm}$. uranium $/ \mathrm{cm}^{2}{ }^{2}$. These were packed in ccntact with 'Kodak' NT.2a plates, which have pre. viously been shown to be capable of recording electron tracks ${ }^{5,6}$. Some of these were shielded with $\frac{3}{8}$ in. of lead and exposed to a flux of $5 \times 10^{11}$ neutrons per $\mathrm{cm} .^{2}$ of plate in the nuclear pile at Harwell.

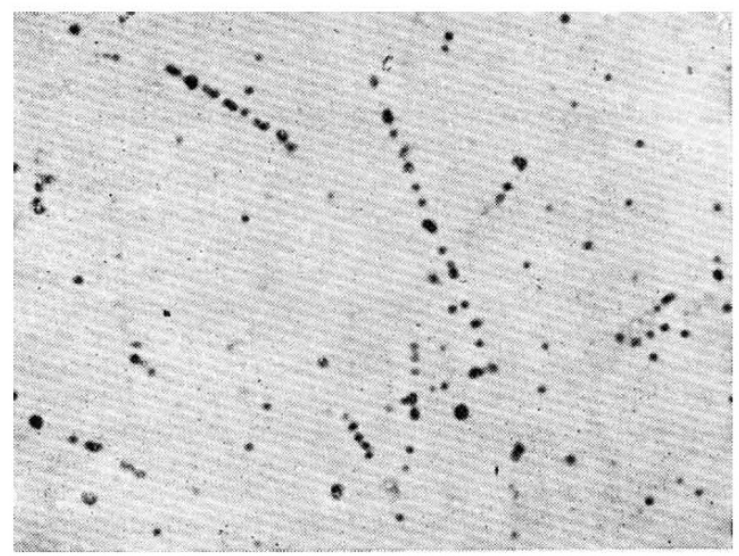

Fig. 2. Tracks given by 40 -min. development in a low-energy para-aminophenol developer (solution I) 